\title{
Intestinal Parasites in Children with Lymphohematopoietic Malignancy in Iran, Mashhad
}

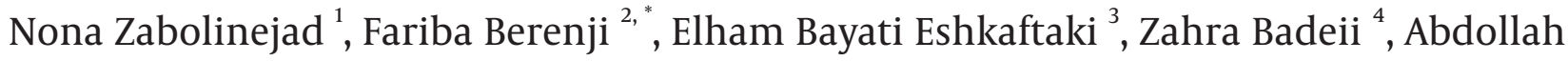 \\ Banihashem ${ }^{4}$, Monavar Afzalaqaei ${ }^{5}$ \\ ${ }^{1}$ Department of Pathology, Mashhad University of Medical Sciences, Mashhad, IR Iran \\ 2 Department of Parasitology \& Mycology, Medical Faculty, Mashhad University of Medical Sciences, Mashhad, IR Iran \\ 3 Department of Parasitology, Shahrekord University of Medical Sciences, Mashhad, IR Iran \\ 4 Department of Pediatrics, Mashhad University of Medical Sciences, Mashhad, IR Iran \\ 5 Department of Biostatistic and Epidemiology, Mashhad University of Medical Sciences, Mashhad, IR Iran \\ ${ }^{*}$ Corresponding author: Fariba Berenji, Department of Parasitology, Mashhad University of Medical Sciences, Mashhad, IR Iran. Tel: +98-5118022235, Fax: +98-5118547255, E-mail: \\ fberenji@yahoo.com.
}

Received: September 29, 2012; Revised: December 22, 2012; Accepted: January 12, 2013

\begin{abstract}
Background: Intestinal parasitic disease can cause serious complications for Immunosuppressed patients.
Objectives: This study determines the prevalence of intestinal parasites in children, with lymphohematopoietic malignancy in Mashhad, Iran.

Patients and Methods: In this cross-sectional study stool samples were collected from 89 children (53 boys, 36 girls) with lymphohematopoitic malignancies under chemotherapy, between the age of 1 and 18 years (mean age 7.5 years). Three fresh stool samples taken for three consecutive days were examined by direct smear, formalin-ether method, trichrome staining and ELISA test for Giardia lamblia coproantigens.

Results: In this study $35.9 \%$ of our patients had parasitic infections and the following parasites were identified; G. lamblia (the most prevalent parasite in children) 16 (18\%), Entamoeba coli 6 (6.7\%) Blastocystis hominis 5(5.6\%) Iodamoeba butschlii 2 (2.2\%). Chilomastics mesnili 1 (1.1\%), Hymenolepis nana 1(1.1\%) and Enterobius vermicularis 1 (1.1\%).

Conclusions: With regards to the high incidence of gasterointestinal parasitic diseases and also because of asymptomatic cases of giardiasis, we recommend evaluation of pediatric patients with malignant lymphohematopoitic disease by at least two different diagnostic methods and three rounds of stool examination in order to prevent possible life threatening outcomes. Coproparasitoscopic study for oncologic patients should be performed and anti-parasitic treatment provided before starting chemotherapy to prevent disseminated parasitic infections. The coproantigen-ELISA is especially advantageous in situations where only a single stool sample can be examined.
\end{abstract}

Keywords: Intestinal Diseases, Parasitic; Hematologic Neoplasms, Children

\section{Background}

Parasitic diseases continue to be a major cause of morbidity and mortality, with more than 3 billion people infected worldwide. Many of these infections occur in the developing world, where improved measures to prevent infection require considerable investment in the public health infrastructure. The segment of the population with significant defects in the immune system continues to grow (1). Intestinal parasites still remain a major public health problem in our country.

\section{Objectives}

Immunocompromised patients have been susceptible to gastrointestinal parasitic infections. In Iran there have been many documents on adults but because of limita- tions regarding malignant children profiles we decided to collect further information on these subjects. The purpose of this cross-sectional study was determining the prevalence of intestinal parasitic infections among lymphohematopoietic malignant children in Mashhad, Iran.

\section{Patients and Methods}

This cross-sectional study was conducted at the hematology-oncology service of Dr Sheikh Children's hospital affiliated to Mashhad University of Medical Sciences, and Parasitology lab of Imam Reza Hospital of Mashhad, from October 2008 to October 2009. The research included all patients between 1 day to 18 years of age with lymphohematopoietic malignancies who were admitted to the hematology oncology department and underwent chemotherapy. Patients who had used antiparasitic or antibiotic

Implication for health policy/practice/research/medical education:

Immunocompromised patients have been susceptible to gastrointestinal parasitic infections. This study determines the prevalence of intestinal parasites in children, with lymphohamatopoietic malignancy.

Copyright (C) 2013, Ahvaz Jundishapur University of Medical Sciences; Licensee Kowsar Ltd. This is an Open Access article distributed under the terms of the Creative Commons Attribution License (http://creativecommons.org/licenses/by/3.0), which permits unrestricted use, distribution, and reproduction in any medium, provided the original work is properly cited. 
drugs were excluded from the study. The protocol was approved by the university's ethical committee. The parents of the patients were interviewed by a nurse to complete a questionnaire on the type of underlying lymphohematopoietic malignancies, clinical symptoms (such as diarrhea, anorexia, abdominal colic, flatulence, fever and weight loss) and duration of treatment, area of residence, contact with animals, source of drinking water, as well as information about the health status of the child.

Stool samples were collected from 89 children with lymphohematopoietic malignancies. Three fresh stool samples taken for three consecutive days were examined by direct smear, formalin-ether method, trichrome staining and ELISA test for Giardia lamblia coproantigens. All specimens were also processed according to the immunoenzymatic assay instruction guide (DRG Giardia Antigen, Germany) to detect G. lamblia specific coproantigen. The results were read using a plate reader with a $450 \mathrm{~nm}$ filter (Awarness, Stat fax 3200). The samples, which yielded a difference in optical density greater than or equal to 0.150 were considered positive, and those with an optical density less than 0.150 were considered negative. Statistical analyses were performed with the Chi square and ANOVA tests. The data were analyzed using the SPSS statistical software ( $11^{\text {th }}$ version). Statistical significance was set at $\mathrm{P}<0.05$.

\section{Results}

Of the 89 patients included in the study, 53 (59.6\%) were male and 36 (40.4\%) female. The subject's age ranged from 1 month to 18 years (mean 7.5 years $S D=3.8$ ). 83 patients had acute lymphoblastic leukemia (ALL), 4 had acute myelogenic leukaemia (AML) and 2 had non-Hodgkin's lymphoma (NHL).

In this study $35.9 \%$ of our patients had parasitic infections and the prevalence of infection according to the type of parasite is shown in Figure 1. The most common parasite was G. lamblia 16 (18\%) and the other parasites were identified as follows: Entamoeba coli 6 (6.7\%), Blastocystis hominis 5 (5.6\%), Iodamoeba butschlii 2 (2.2\%). Chilomastics mesnili 1 (1.1\%), Hymenolepis nana 1(1.1\%) and Enterobius vermicularis 1 (1.1\%). The age group of 7-8 years showed maximum frequency of Giardia infection, followed by 5 - 6 years. Although the frequency of Giardia was more in females than in males, there was no significant difference between them $(\mathrm{P}=0.144) .64 .3 \%$ of patients with giardiasis had no symptoms and $35.7 \%$ had clinical symptoms. The most common clinical symptoms were as follows: recurrent abdominal discomfort (35.7\%), diarrhea (16.3\%), flautness (15.8\%), weight loss (50\%) and anorexia (10\%).

In this study some factors such as water supply and contact with animals was documented, our findings showed that $44.4 \%$ of children with giardiasis use unsanitary water supply (wells or water containers) and $22.2 \%$ had contact with animals. 14 cases $(87.5 \%)$ of giardiasis were detected with the ELISA method. The sensitivity and spec- ificity of this method were $98.6 \%$ and $81.3 \%$, respectively.

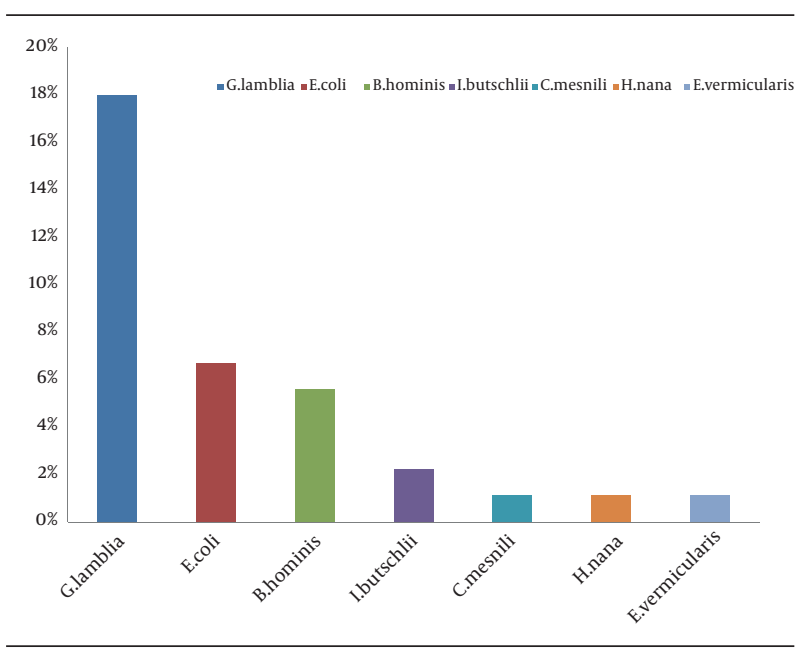

Figure 1. The Prevalence Rate of the Parasitic Infections According to Species of Parasite in Children, with Lymphohematopoietic Malignancy in Mashhad, Iran.

\section{Discussion}

Parasitic gastrointestinal infections have been variably reported among immune compromised adults in Iran while data on children have been limited. This prospective cross-sectional study aimed to assess the clinical profile of intestinal parasitic infections among lymphohematopoietic malignant children in Mashhad, Iran. Our study demonstrated a prevalence of $35.9 \%$ for parasitic infection in lymphohematopoietic malignant children. In Turkey, Aksoy et al. found parasitic infections in $42 \%$ of children with neoplasm (1) but in Mexico City RiveraLuna et al. found parasitosis in $12.9 \%$ of childhood acute leukemia (2).

In India, Rudrapatna et al. in a retrospective study found intestinal parasitic infections in $16.5 \%$ of patients with malignancy (3). Menon et al. found intestinal parasites in $42 \%$ of a group of Malaysian children with cancer (4). Our study shows that the high prevalence rate of parasitic infections may be due to three factors: first, the type of malignancy (lymphohematopoietic malignancies versus other malignant neoplasms); second, immunocompromising effect of the chemotherapy for cancer (all the patients in our study underwent chemotherapy), and third (the most important factor), use of two different diagnostic methods (ELISA in addition to microscopic examination) which led to a higher sensitivity for detection of intestinal parasites.

According to our findings, $18 \%$ of patients had giardiasis so that Giardia is the most frequently identified enteric parasite in our study; Aksoy et al. found giardiasis in 14\% of children with neoplasm (1). However, Rudrapatna et al. reported giardiasis for 3.1\% of malignant patients (3). Infection with G. lambliais caused by ingestion of food or water contaminated with cysts. It has worldwide distri- 
bution and occurs in developed and developing country (5). G. lamblia transmission can be from person to person but is more commonly waterborne, a result of the relative resistance of G. lamblia cysts to chlorination. Clinical features of giardiasis vary from the asymptomatic carrier to a severe malabsorption syndrome. Many factors could affect this variation of clinical effects like virulence of the Giardia strain, age, host immune system condition and number of cysts swallowed (6).

$64.3 \%$ of our patients with giardiasis had no symptoms and $35.7 \%$ had clinical symptoms. The most common clinical symptoms were recurrent abdominal discomfort (35.7\%), diarrhea (16.3\%), flautness (15.8\%), weight loss (50\%), and anorexia (10\%). Although, in our study giardiasis was more frequent in females (56.25\%) than males (43.75\%) it was not statistically significant $(P=0.144)$.

The age group of 7-8 years showed maximum frequency of Giardia infection, followed by 5-6 years. This infection is particularly high in poor and developing countries due to the use of contaminated drinking water, inadequate sanitary conditions and poor personal hygiene (7). In this study, some factors such as water supply and contact with animals have been documented; our findings show that $44.4 \%$ of children with giardiasis use unsanitary water supply (wells or water containers) and also $22.2 \%$ of children with giardiasis had contact with animals that shows unsanitary conditions. This may indicate that immune compromised children and their parents need health education about prevention of parasitic infections and must also have access to sanitary conditions and good water supply.

Routine stool examinations are normally recommended for the recovery and identifications of intestinal protozoa. However, in the case of G. lamblia, because the organisms are attached so securely to the mucosa by the means of a sucking disk, a series of five or six equal stool samples may be examined without recovering the organisms. The organisms also tend to be passed in the stool on a cyclical basis (8). The most important thing about these parasites is that the appearance of Giardia in the stool is not so regular and frequent tests are required in order to detect cyst shedding and one direct smear that uses small amounts of sample cannot detect Giardia parasites; therefore we need three rounds of stool examination that can increase the chances of diagnosis.

In our study three fresh stool samples taken for three consecutive days were examined by three methods for diagnosis of parasitic infections; direct smear, formalin ether method, trichrome staining and ELISA test for G. lamblia coproantigens. Many serological assays have been introduced to detect serum antibodies but because of the lack of appropriate antigens of Giardia, the sensitivity of serological tests remains poor. Besides, detection of coproantigen has been successful and commercial kits are available which have been reported to be more sensitive than microscopic diagnosis of giardiasis (9). Pro- cedures involving the enzyme-linked immunosorbent assay (ELISA) have also been developed to detect Giardia antigen in feces. The ELISA is at least as sensitive as microscopic wet examinations (8).Comparison of ELISA test and formalin ether in our study shows that from 16 cases of giardiasis 14cases can be detected with the ELISA method. The sensitivity and specificity of this method was $98.6 \%$ and $81.3 \%$, respectively.

In this study, formalin - ether test considered as a gold standard test and other parasitologic diagnostic methods like direct method and trichrome staining were used and ultimately ELISA for G. lamblia antigen detection was tested. It is known that fecal examination to detect G. lamblia cysts or trophozoites produces a high percentage of false-negative results. Rocha et al. showed that the assay was able to identify all 30 positive patients (sensitivity = $100.0 \%$ ). The assay seems to be a good alternative for giardiasis diagnosis, especially when the fecal examination was repeatedly negative and the patient presents symptoms similar to that of giardiasis (10).

The coproantigen-ELISA is especially advantageous in situations where only a single stool sample can be examined. It should not, however, replace microscopic examination of stool specimens for ova and parasites since other potential pathogens would otherwise escape (11). Routine microscopic detection of parasite is inexpensive, but needs expert technicians. Methods for antigen detection can be carried out more quickly and without proficient technicians (12). We highlight the importance of diagnosis and the skills of the laboratory of parasitology, since most parasitic infections responsible for diarrhea in lymphohematopoietic malignant children can be treated. We conclude that because of irregular shedding of Giardia and time consuming methods for detection of Giardia cysts and trophozoites, it is recommended to use sensitive methods like fecal antigen detection instead of routine methods.

\section{Acknowledgements}

We thank Mr. Majid Ganjbakhsh for his kind efforts during this project and technical help.

\section{Authors' Contribution}

Study concept and design: Nona Zabolinejad, Fariba Berenji. Zahra Badeii, Abollah Banihashem. Analysis and interpretation of data: Elham Bayati Eshkaftaki, Fariba Berenji, Monavar Afzalaqaei. Drafting of the manuscript: Fariba Berenji. Critical revision of the manuscript for important intellectual content: Fariba Berenji. Statistical analysis: Monavar Afzalaqaei.

\section{Financial Disclosure}

Authors declare that there are not any relevant financial interests related to the material in the manuscript. 


\section{Funding/Support}

The authors greatly acknowledge the Research Council of Mashhad University of Medical Sciences (MUMS), Mashhad, Iran for their financial grant. The results presented in this work have been taken from Elham Bayati Eshkaftaki thesis, with the ID number "258."

\section{References}

1. Aksoy U, Erbay A, Akisu C, Apa H, Ozkoc S, Ozturk S. Intestinal parasites in children with neoplasms. Turk J Pediatr. 2003;45(2):12932.

2. Rivera-Luna R, Cardenas-Cardos R, Martinez-Guerra G, Ayon A, Leal C, Rivera-Ortegon F. Childhood acute leukemia and intestinal parasitosis. Leukemia.1989;3(11):825-6.

3. Rudrapatna JS, Kumar V, Sridhar H. Intestinal parasitic infections in patients with malignancy.J Diarrhoeal Dis Res. 1997;15(2):71-4.

4. Menon BS, Abdullah MS, Mahamud F, Singh B. Intestinal parasites in Malaysian children with cancer. J Trop Pediatr. 1999;45(4):241-2.

5. Stark D, Barratt JL, van Hal S, Marriott D, Harkness J, Ellis JT. Clinical significance of enteric protozoa in the immunosuppressed human population. Clin Microbiol Rev. 2009;22(4):634-50.

6. Rosales-Borjas DM, Diaz-Rivadeneyra J, Dona-Leyva A, ZambranoVilla SA, Mascaro C, Osuna A, et al. Secretory immune response to membrane antigens during Giardia lamblia infection in humans. Infect Immun. 1998;66(2):756-9.

7. Ahmed AK, Malik B, Shaheen B, Yasmeen G, Dar JB, Mona AK, et al. Frequency of intestinal parasitic infestation in children of 5-12 years of age in Abbottabad. J Ayub Med Coll Abbottabad. 2003;15(2):28-30.

8. Garcia LS, Bruckner DA. Diagnostic medical parasitology. American Society for Microbiology (ASM); 1997. ISBN 1555811167.

9. Faubert G. Immune response to Giardia duodenalis. Clin Microbiol Rev. 2000;13(1):35-54.

10. Rocha MO, Mello RT, Guimaraes TM, Toledo VD, Moreira MD, Costa CA. Detection of a giardia lamblia coproantigen by using a commercially available immunoenzymatic assay, in belo horizonte, brazil. Rev Inst Med Trop Sao Paulo. 1999;41(3):151-4.

11. Jelinek T, peyerl G, Loscher T, Nothdurft HD. Giardiasis in travellers: evaluation of an antigen-capture ELISA for the detection of Giardia lamblia-antigen in stool. Z Gastroenterol. 1996;34(4):23740.

12. Uyar Y, Taylan Ozkan A. [Antigen detection methods in diagnosis of amebiasis, giardiasis and cryptosporidiosis]. Turkiye Parazitol Derg. 2009;33(2):140-50. 